\title{
INDUCTION AND RESTRICTION AS ADJOINT FUNCTORS ON REPRESENTATIONS OF LOCALLY COMPACT GROUPS
}

\section{ROBERT A. BEKES}

Department of Mathematics Santa Clara University Santa Clara, CA 95053 and

PETER J. HILTON

Department of Mathematical Sciences

State University of New York at Binghamton Binghamton, NY 13902-6000

(Received April 16, 1992)

\begin{abstract}
In this paper the Frobenius Reciprocity Theorem for locally compact groups is looked at from a category theoretic point of view.
\end{abstract}

KEY WORDS AND PHRASES. Locally compact group, Frobenius Reciprocity, category theory.

1991 AMS SUBJECT CLASSIFICATION CODES. 22D30, 18A23

\section{INTRODUCTION.}

In [1] C. C. Moore proves a global version of the Frobenius Reciprocity Theorem for locally compact groups in the case that the coset space has an invariant measure. This result, for arbitrary closed subgroups, was obtained by A. Kleppner [2], using different methods. We show how a slight modification of Moore's original proof yields the general result. It is this global version of the reciprocity theorem that is the basis for our categorical approach. (See [3] for a description of the necessary category-theoretical concepts.)

We begin by setting up the machinery necessary to discuss the reciprocity theorem. Next we show how, using the global version of the theorem, the functors of induction and restriction are adjoint. The proofs of these results are at the end of the paper.

\section{THE MAIN RESULTS.}

Throughout $G$ is a separable locally compact group and $K$ is a closed subgroup. Let $G / K$ denote the space of right cosets of $K$ in $G$ and for $s \in G$, we write $\bar{s}$ for the coset $K s$. Let $\mu$ be a quasi-invariant measure on $G / K$, see [4]. Then there exists a continuous positive function $R$ on $G / K \times G$ such that

$$
\int_{G / K} f\left(\bar{s} t^{-1}\right) d \mu(\bar{s})=\int_{G / K} f(\bar{s}) R(\bar{s}, t) d \mu(\bar{s})
$$

for all $t \in G$ and all compactly supported continuous functions $f$ on $G / K$. It is by 
exploiting the function $R$ that we are able to avoid requiring an invariant measure on $G / K$.

Let $\rho$ be a strongly continuous unitary representation of $K$ on the Hilbert space $H(\rho)$. Then if $f$ is a function from $G$ to $H(\rho)$ such that $f(k s)=\rho(k) f(s)$ for all $k \in K$ and $s \in G$, the function $s \rightarrow\|f(s)\|$ is constant on the cosets of $K$ in $G$. Therefore, as in [1], we define $H(\operatorname{Ind} \rho)$ to be the Banach space of such functions which are weakly measurable and for which

$$
\int_{G / K}\|f(s)\| d \mu(\bar{s})<\infty .
$$

For $t \in G$ define the operator $\operatorname{Ind} \rho(t)$ on $H(\operatorname{Ind} \rho)$ by $\operatorname{Ind} \rho(t) f(s)=f(s t) R(\bar{s}, t)$. From (1) we see that $\operatorname{Ind} \rho(t)$ is an isometry. That $t \rightarrow \operatorname{Ind} \rho(t)$ is a representation of $G$ follows from the cocycle identity, $\quad R\left(\bar{s}, t_{1} t_{2}\right)=R\left(\bar{s} t_{1}, t_{2}\right) R\left(\bar{s}, t_{1}\right)$. Note that this is the summable induced representation used by Moore in his result [1] except that, as indicated, we have included the function $R$ to compensate for the lack of an invariant measure on $G / K$. It is easy to see that Ind is a functor from $\mathcal{C}$ to $\mathfrak{T}$, where these categories are now described.

Let $\mathscr{I}$ be the category whose objects consist of continuous representations of $G$ and 'morphisms, $\mathscr{T}\left(\pi_{1}, \pi_{2}\right)$, the intertwining operators. That is, if $\pi_{1}$ and $\pi_{2}$ belong to $\mathscr{T}$, a morphism $T \in \mathscr{I}\left(\pi_{1}, \pi_{2}\right)$ is a continuous operator $T: H\left(\pi_{1}\right) \rightarrow H\left(\pi_{2}\right)$ such that $\pi_{2}(s) T=T \pi_{1}(s)$, for all $s \in G$. It follows that, for fixed $\pi_{1}, \pi_{2}, \mathscr{D}_{(}\left(\pi_{1}, \pi_{2}\right)$ is a Banach space. Let $\mathrm{C}$ be the category of continuous unitary representations of $K$ and associated morphisms, $\mathcal{C}\left(\rho_{1}, \rho_{2}\right)$, which are again the intertwining operators.

Let $\pi$ be a continuous unitary representation of $G$. Then Res $\pi$, the restriction of $\pi$ to $K$, belongs to $\mathrm{C}$, and Res may be viewed as a functor from $\mathfrak{D}$ to $\mathrm{e}$. Let $B \in \mathcal{C}(\rho, \operatorname{Res} \pi)$ and, for $f \in \operatorname{Ind} \rho$, define, as in [1],

by the rule

$$
\eta: \mathcal{C}(\rho, \operatorname{Res} \pi) \rightarrow \mathscr{I}(\operatorname{Ind} \rho, \pi)
$$

$$
\eta(B) f=\int_{G / K} \pi^{*}(s) B f(s) d \mu(\bar{s}) .
$$

It is shown in [1] that $\eta(B)$ is a bounded linear map from $H(\operatorname{Ind} \rho)$ to $H(\pi)$. Now let $t \in G$ and $\xi \in H(\pi)$, then

$$
\begin{aligned}
\langle\pi(t) \eta(B) f, \xi\rangle \quad & =\left\langle\pi(t) \int_{G / K} \pi^{*}(s) B f(s) d \mu(\bar{s}), \xi\right\rangle=\left\langle\int_{G / K} \pi^{*}(s) B f(s) d \mu(\bar{s}), \pi^{*}(t) \xi\right\rangle \\
& =\int_{G / K}\left\langle\pi^{*}(s) B f(s), \pi^{*}(t) \xi\right\rangle d \mu(\bar{s})=\int_{G / K}\left\langle\pi(t) \pi^{*}(s) B f(s), \xi\right\rangle d \mu(\bar{s}) \\
& =\int_{G / K}\left\langle\pi^{*}\left(s t^{-1}\right) B f(s), \xi\right\rangle d \mu(\bar{s})=\int_{G / K}\left\langle\pi^{*}(s) B f(s t), \xi\right\rangle R(\bar{s}, t) d \mu(\bar{s})
\end{aligned}
$$




$$
\begin{aligned}
& =\left\langle\int_{G / K} \pi^{*}(s) B f(s t) R(\bar{s}, t) d \mu(\bar{s}), \xi\right\rangle=\left\langle\int_{G / K} \pi^{*}(s) B[\operatorname{Ind} \rho(t) f](s) d \mu(\bar{s}), \xi\right\rangle \\
& =\langle\eta(B) \operatorname{Ind} \rho(t) f, \xi\rangle .
\end{aligned}
$$

Thus $\pi(t) \eta(B)=\eta(B) \operatorname{Ind} \rho(t)$, so that $\eta(B) \in \mathscr{T}(\operatorname{Ind} \rho, \pi)$.

The global version of the Frobenius Reciprocity Theorem in this setting is

Theorem 1. The map $\eta$ is an isometric isomorphism of $\mathcal{C}(\rho, \operatorname{Res} \pi)$ onto $\mathfrak{T}(\operatorname{Ind} \rho, \pi)$.

We will show, further, that $\eta$ is a natural adjunction. We first make explicit how Ind acts on a morphism $\zeta \in \mathbb{e}\left(\rho_{1}, \rho_{2}\right)$. Let $f \in H\left(\operatorname{Ind} \rho_{1}\right)$. Since $\zeta$ is continuous, the function $s \rightarrow \zeta(f(s))=[(\operatorname{Ind} \zeta) f](s)$ is measurable and

$$
\int_{G / K}\|\zeta(f(s))\| d \mu(\bar{s}) \leq\|\zeta\| \int_{G / K}\|f(s)\| d \mu(\bar{s})<\infty .
$$

Moreover, for $k \in K$,

$$
[(\operatorname{Ind} \zeta) f](k s)=\zeta(f(k s))=\zeta\left(\rho_{1}(k) f(s)\right)=\rho_{2}(k) \zeta(f(s))=\rho_{2}(k)[(\operatorname{Ind} \zeta) f](s)
$$

Thus $(\operatorname{Ind} \zeta) f \in H\left(\operatorname{Ind} \rho_{2}\right)$. It is plain that $\operatorname{Ind}\left(\zeta \zeta^{\prime}\right)=(\operatorname{Ind} \zeta)\left(\operatorname{Ind} \zeta^{\prime}\right)$, so that Ind is, as claimed, a functor.

Now the naturality of the adjunction $\eta$ is expressed by the following relation.

Theorem 2. Let $\zeta \in \mathrm{C}\left(\rho_{1}, \rho_{2}\right)$ and $\psi \in \mathscr{T}\left(\pi_{2}, \pi_{1}\right)$. Then, for all $B \in \mathcal{C}\left(\rho_{2}, \operatorname{Res} \pi_{2}\right)$,

$$
\psi \circ \eta(B) \circ \operatorname{Ind} \zeta=\eta(\operatorname{Res} \psi \circ B \circ \zeta) \text {. }
$$

\section{PROOFS.}

Moore's proof [1] of Theorem 1 , where $G / K$ has an invariant measure, is easily modified to extend to arbitrary closed subgroups $K$ as follows:

Proof Modification: Let $\tau: G / K \rightarrow G$ be a Borel cross section. Then in [1] the set $S$ is just the image of $\tau$ and $\mu$ can be regarded as a measure on $S$. It follows that $H(\operatorname{Ind} \rho)$ is isomorphic with $L_{1}(S, \mu, H(\rho))$, the space of $\mu$-measurable $L_{1}$-functions from $S$ into $H(\rho)$, see [4]. Our argument remains the same as [1] until we have to show that the map $\eta$ is surjective. 
So let $C \in \mathscr{D}(\operatorname{Ind} \rho, \pi)$. Then for $g \in L_{1}(S, \mu, H(\rho))$, it is still true that

$$
C g=\int_{S} \pi^{*}(s) B(s) g(s) d \mu(s)
$$

where $\quad s \rightarrow B(s): H(\rho) \rightarrow H(\pi) \quad$ is a measurable, operator valued function. We need to show that $B(s)$ is equal to a constant $B$ almost everywhere and that $B \in \mathfrak{C}(\rho, \operatorname{Res} \pi)$. It is this step that requires some minor change.

In his proof Moore defines two Borel maps $k(s, t)$ and $\ell(s, t)$ from $S \times G$ to $K$ and $S$ respectively to have the properity that $s t=k(s, t) \ell(s, t)$. These maps can be written in terms of $\tau$ as follows: $\ell(s, t)=\tau(\bar{s} t)$ and $k(s, t)=\tau(\bar{s}) t \tau(\bar{s} t)^{-1}$. It is easier to work with the map $\tau$ and then to translate back to $k$ and $\ell$.

Rewriting (3) using $\tau$ we get

$$
C g=\int_{S} \pi^{*}(\tau(\bar{s})) B(\tau(\bar{s})) g(\tau(\bar{s})) d \mu(s)
$$

Following [1], for $t \in G$,

$$
\pi\left(t^{-1}\right) C g=\int_{S} \pi^{*}(\tau(\bar{s}) t) B(\tau(\bar{s})) g(\tau(\bar{s})) d \mu(s)
$$

and

$$
\left[\operatorname{Ind} \rho\left(t^{-1}\right) g\right](s)=\rho\left(\tau(\bar{s}) t^{-1} \tau\left(\bar{s} t^{-1}\right)^{-1}\right) g\left(\tau\left(\bar{s} t^{-1}\right)\right) R\left(\bar{s}, t^{-1}\right)
$$

Substituting (6) into (4) gives

$$
C\left[\operatorname{Ind} \rho\left(t^{-1}\right) g\right]=\int_{S} \pi^{*}(\tau(\bar{s})) B(\tau(\bar{s})) \rho\left(\tau(\bar{s}) t^{-1} \tau\left(\bar{s} t^{-1}\right)^{-1}\right) g\left(\tau\left(\bar{s} t^{-1}\right)\right) R\left(\bar{s}, t^{-1}\right) d \mu(s) .
$$

Making the change of variables $s \rightarrow s t \quad$ we get

$$
\left.C\left[\operatorname{Ind} \rho\left(t^{-1}\right) g\right]=\int_{S} \pi^{*}(\tau(\bar{s} t)) B(\tau(\bar{s} t)) \rho\left(\tau(\bar{s} t) t^{-1} \tau(\bar{s})^{-1}\right) g(\tau(\bar{s}))\right) R\left(\bar{s} t, t^{-1}\right) R\left(\bar{s}, t^{-1}\right) d \mu(s) .
$$

By the cocycle identity $R\left(\bar{s} t, t^{-1}\right) R\left(\bar{s}, t^{-1}\right)=1$. Therefore

$$
\left.C\left[\operatorname{Ind} \rho\left(t^{-1}\right) g\right]=\int_{S} \pi^{*}(\tau(\bar{s} t)) B(\tau(\bar{s} t)) \rho\left(\tau(\bar{s} t) t^{-1} \tau(\bar{s})^{-1}\right) g(\tau(\bar{s}))\right) d \mu(s) .
$$

Equating the expression for $\pi\left(t^{-1}\right) C g$ in (5) with that for $C\left[\operatorname{Ind} \rho\left(t^{-1}\right) g\right]$ in (7) we get, for almost all $s \in S$ and $t \in G$,

$$
\pi^{*}(\tau(\bar{s}) t) B(\tau(\bar{s}))=\pi^{*}(\tau(\bar{s} t)) B(\tau(\bar{s} t)) \rho\left(\tau(\bar{s} t) t^{-1} \tau(\bar{s})^{-1}\right)
$$

Rewriting this, using the fact that $\pi$ is unitary, yields

$$
\pi\left(\tau(\bar{s} t) t^{-1} \tau(\bar{s})^{-1}\right) B(\tau(\bar{s}))=B(\tau(\bar{s} t)) \rho\left(\tau(\bar{s} t) t^{-1} \tau(\bar{s})^{-1}\right)
$$


Now let $s=\tau(\bar{s})$. Then we have

$$
\pi^{*}(k(s, t)) B(s)=B(\ell(s, t)) \rho^{*}(k(s, t)),
$$

which is $(*)$ in [1], and the rest of the proof remains unchanged.

We now move to the proof of Theorem 2.

Proof: Starting with the left side of the equality, let $\xi \in \pi_{1}$ and $f \in \operatorname{Ind} \rho_{1}$ then

$$
\begin{aligned}
\langle[\psi \circ \eta(B) \circ \operatorname{Ind} \zeta] f, \xi\rangle & =\left\langle\psi \int_{G / K} \pi_{2}{ }^{*}(s) B(\zeta(f(s)) d \mu(\bar{s}), \xi\rangle\right. \\
& =\left\langle\int_{G / K} \pi_{2}{ }^{*}(s) B\left(\zeta(f(s)) d \mu(\bar{s}), \psi^{*} \xi\right\rangle\right. \\
& =\int_{G / K}\left\langle\pi_{2}{ }^{*}(s) B\left(\zeta(f(s)), \psi^{*} \xi\right\rangle d \mu(\bar{s})\right. \\
& =\int_{G / K}\left\langle(\operatorname{Res} \psi) \pi_{2}{ }^{*}(s) B(\zeta(f(s)), \xi\rangle d \mu(\bar{s})\right. \\
& =\int_{G / K}\left\langle\pi_{1}{ }^{*}(s)(\operatorname{Res} \psi) B(\zeta(f(s)), \xi\rangle d \mu(\bar{s})\right. \\
& =\left\langle\int_{G / K} \pi_{1}{ }^{*}(s)(\operatorname{Res} \psi) B(\zeta(f(s)) d \mu(\bar{s}), \xi\rangle\right. \\
& =\langle\eta(\operatorname{Res} \psi \circ B \circ \zeta) f, \xi\rangle .
\end{aligned}
$$

Since $f$ and $\xi$ were arbitrary, $\eta(\operatorname{Res} \psi \circ B \circ \zeta)=\psi \circ \eta(B) \circ \operatorname{Ind} \zeta$, as claimed.

Let us close with one consequence of the adjointness relation.

Corollary 3. Let $\zeta: \rho_{1} \rightarrow \rho_{2}$ be surjective. Then if $\eta(B) \circ \operatorname{Ina} \zeta=0, B=0$.

Proof: For if $\eta(B) \circ \operatorname{Ind} \zeta=0$, then $\eta(B \circ \zeta)=0, \quad$ so $B \circ \zeta=0$. But $\zeta$ is surjective, so $B=0$.

Notice that the adjointness relation expressed in Theorem 2 must be very carefully exploited. For Induction is only defined on unitary representations, and produces continuous representations, whereas Restriction can be defined on unitary or continuous representations. Thus $\eta$ itself converts unitary intertwining operators into continuous intertwining operators. We hope to examine this feature of adjunction in a subsequent note, together with the enrichment implicit in Theorem 1, where the morphism sets of $C$ and $\mathscr{D}$ have the structure of Banach spaces and $\eta$ is enriched to an isometric isomorphism (and not merely a bijection). 


\section{REFERENCES}

1. C. C. Moore, On the Frobenius reciprocity theorem for locally compact groups, Pacific Journal of Mathematics, 12 (1962), 359-365.

2. A. Kleppner, Intertwining forms for summable induced representations, Transactions of the American Mathematical Society, 112 (1964), 164 - 183.

3. H. B. Griffiths and P. Hilton, A Comprehensive Textbook of Classical Mathematics, Springer - Verlag, New York, 1970.

4. H. Reiter, Classical Harmonic Analysis and Locally Compact Groups, Oxford University Press, London, 1968. 


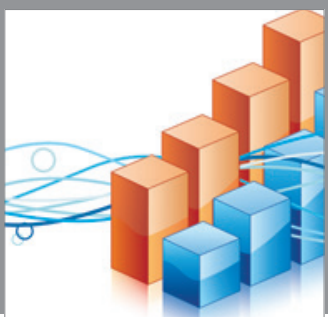

Advances in

Operations Research

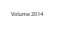

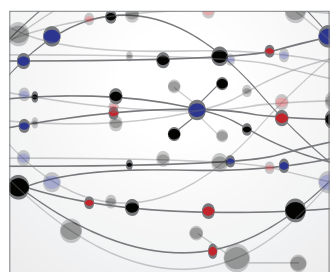

\section{The Scientific} World Journal
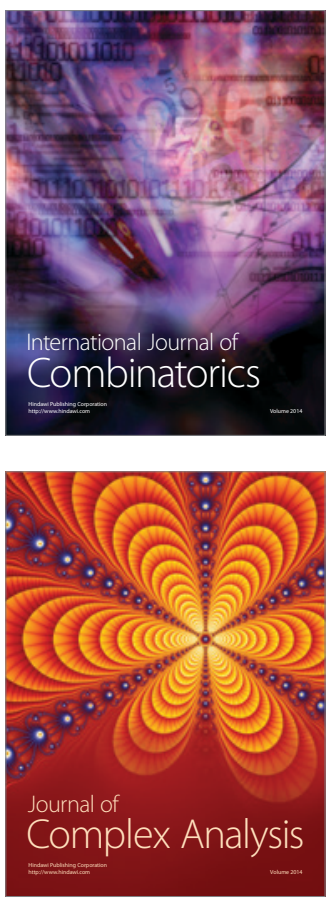

International Journal of

Mathematics and

Mathematical

Sciences
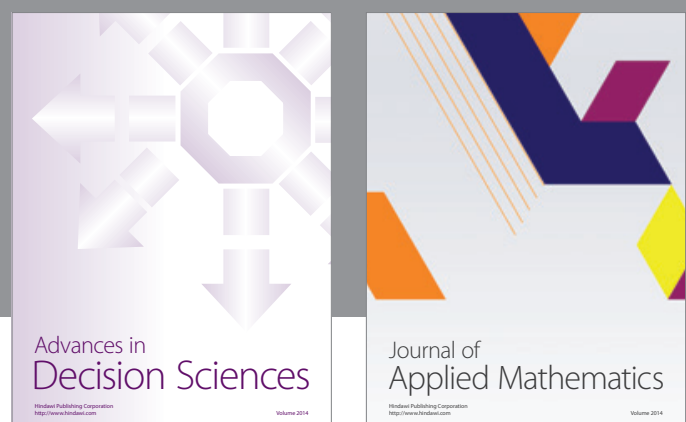

Journal of

Applied Mathematics
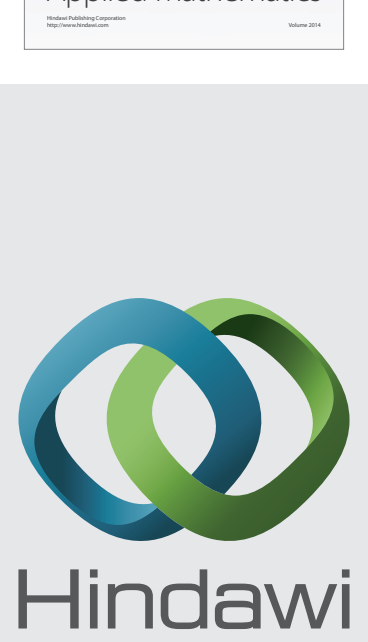

Submit your manuscripts at http://www.hindawi.com
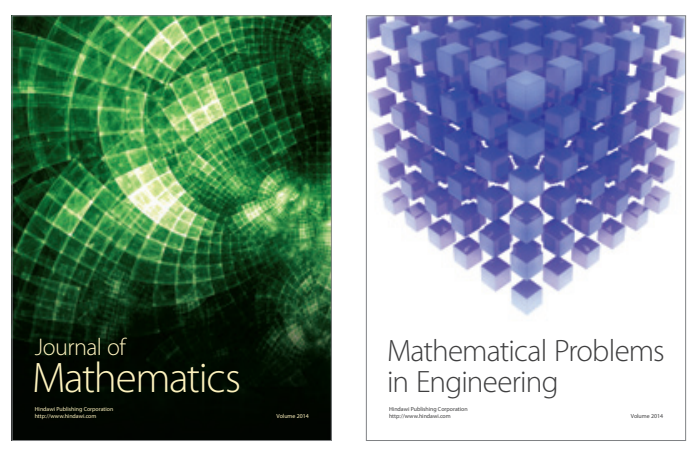

Mathematical Problems in Engineering
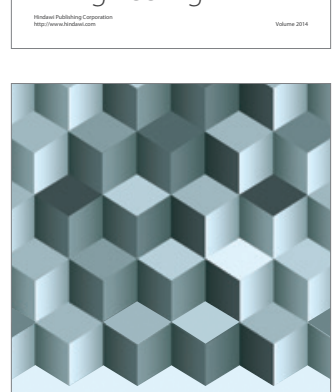

Journal of

Function Spaces
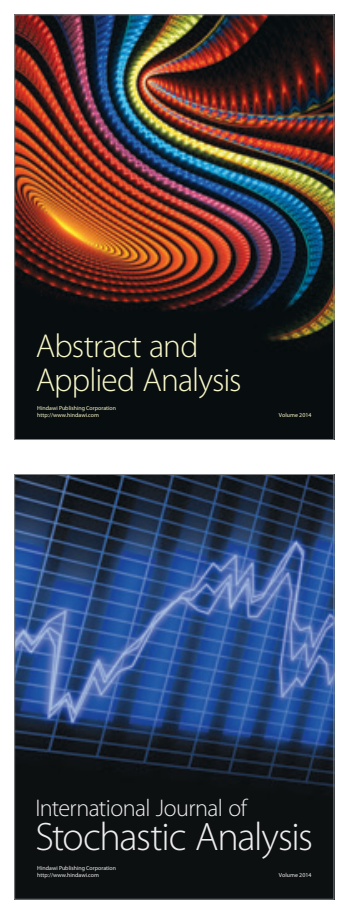

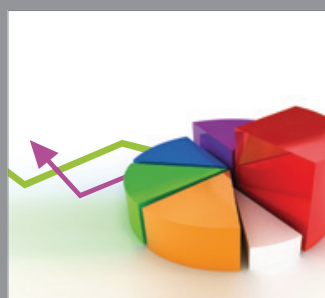

ournal of

Probability and Statistics

Promensencen
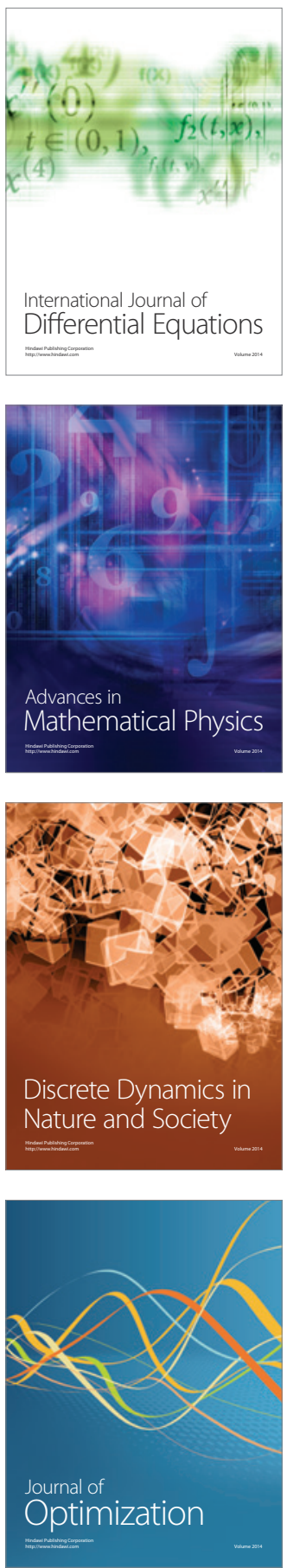